\title{
Research Paper \\ Identifying and Prioritizing Effective Factors in Creating Rural Enterprises Clusters in Choram Township
}

\author{
Marziye Erjaee ${ }^{1},{ }^{*}$ Homayoon Moradnezhadi², Mohamad Salavarzi ${ }^{3}$, Yarmohamad Ghasemi ${ }^{4}$
}

1. MSc., Department of Entrepreneurship and Rural Development, Faculty of Agriculture, Ilam University, Ilam, Iran.

2. Assistant Professor, Department of Entrepreneurship and Rural Development, Faculty of Agriculture, Ilam University, Iran.

3. Assistant Professor, Department of Architectural, Faculty of Engineering, llam University, Ilam, Iran.

4. Associate Professor, Department of Social Sciences, Faculty of Literature and Humanities, Ilam University, Ilam, Iran.

Citation: Erjaee, M., Moradnezhadi, H., Salavarzi, M., \& Ghasemi, Y. (2016). Identifying and Prioritizing Effective Factors in Creating Rural Enterprises Clusters in Choram Township. Journal of Rural Research, 7(3), 504-515. http://dx.doi.org/10.21859/jjr-07036

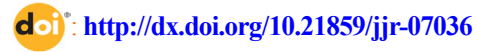

Received: 19 Apr. 2016 Accepted: 1 Jul. 2016

Keywords:

Analytic hierarchy process, Business cluster, Choram township, Rural business

\begin{abstract}
A B STRACT
The main goal of the current study was to identify and prioritize effective factors in creating enterprise clusters in Choram county using analytic hierarchy process (AHP). A descriptive-survey method was used for the study. Statistical population included experts who had field experience in the business clusters in Choram county. Purposeful sampling method was used and finally 44 experts were selected to complete the questionnaires. The instrument used for collecting data was structured interview using Al Saati nine order professional questionnaire. The validity of the questionnaire was determined by the expert opinions of the university professors and experts. The reliability of the questionnaire was assured by considering incompatibility of decision matrices due to the judgment of each respondent. The statistical software, SPSS 23 and Expert Choice 11 were used for analyzing data. According to considered criteria in this research, economic factor was the most important factor and infrastructure factor was the least important criterion for creating business clusters in Choram County.
\end{abstract}

\section{Extended Abstract}

\section{Introduction}

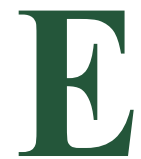

conomists have already found that special locations are suitable for special activities and also companies involved in same or related activities intend to cluster. Profiting of industrial clusters has been known as one of the most important and successful industrial and regional approaches that has attracted policy makers of developing and developed countries. An industrial cluster includes commercial and non-commercial institutions concentrated in a geographical and economic region that make horizontal and vertical relationships for producing one or more same final product(s) and despite competition, they do collective work and have common measures in most cases. Business clusters, besides producer firms that are the main cluster activists, include suppliers, buyers, sub-contractors, exporters, machine tools and parts, various supply institutions, and public services that facilitate directly and or indirectly production in a cluster. Furthermore, it also includes other groups of activists such as unions and associations that are business encouragers in various parts. According to this idea, business clusters in rural regions can have economic effects (such as employment, producing products, delivery services, cooperation and network, added value, and multiplier effect) social effects (such as interaction with the community, local

\section{* Corresponding Author:}

Homayoon Moradnezhadi, PhD

Address: Department of Entrepreneurship and Rural Development, Faculty of Agriculture, Ilam University, Ilam, Iran. Tel: +98 (912) 5856830

E-mail: homayoon.moradnezhadi@ilam.ac.ir 
and community development, providing training opportunities, and the last support and supply), and environmental effects (such as environmental awareness and environment protection). Internal communications of these institutes will lead to decrease in costs and facilitate access to the inputs, production knowledge and technology, sale markets, and costumers' needs. Because in business clustering, the importance of each effective factors is different from one region to another, recognition of these factors is essential and the most important issues that planners need. Therefore, the main goal of the current study was to identify and prioritize effective factors on creating enterprise clusters in Choram County using hierarchy analytic process.

\section{Methodology}

Analytic hierarchy process (AHP) is one of the decision making methods with multi-index that is applied to compare various options. This method was invented by Tomas L Saaty in 1980. This research is practical with respect to its goal, and a field research with respect to the control level of parameters. It is a descriptive research based on data monitoring and it will be profited from mensuration besides the descriptive methods. Required data in this research were collected via structured interview by using Tomas L Saaty questionnaire. Statistical population included managers and planners, employers of industrial estate, technical experts, and main producers and products exporters in Choram Township that targeted sampling method was used because of the wide range of the research. The criteria were obtained using review references and interview with province planning managers. Accordingly, criteria of economic, environmental, government infrastructures, and strategic factors and then sub-criteria were identified. Then the hierarchy of the research subject was designed and accordingly this questionnaire was provided. After design, the validity of questionnaire was confirmed by specialist opinions of one instructor and two regional experts. By considering the incompatibility of the decision matrices obtained from judgment of each responder, we were assured of the stability of obtained results from questionnaire. In the next step, a list of experts (who are active in Choram Township) with high education was prepared. Finally, 45 experts were selected for completing the questionnaires. The obtained results were analyzed using Expert Choice 11 software.

\section{Results}

According to the collected information, the mean age of the responders was 37 years old that $73.3 \%$ of them were men and $26.3 \%$ were women. Furthermore, the youngest and oldest responders were 27 and 57 years old, respectively. About $13.2 \%$ of the studied people were graduated in associate degree, $47.4 \%$ in bachelor and $39.5 \%$ in masters and $\mathrm{PhD}$ degrees. The education range of them fluctuated from associate degree to $\mathrm{PhD}$. Comparing result of various factors showed that the economic criterion had the maximum score $(0.365)$ and so the maximum effect on clustering in the region. The governmental strategy and environmental factors were in the next rank, respectively and infrastructure criterion was located in last rank with score of 0.120 . Therefore, the criteria of economic and infrastructure had the maximum and minimum significance for creating business clusters in Choram Township.

\section{Discusion}

Therefore, the criteria of economic and infrastructure had the maximum and minimum significance for creating business clusters in Choram Township. Incompatibility rate of bigeminal criteria comparisons was 0.6 compared to the target value that was smaller than 0.1 . Thus, the compatibility of matrices of the bigeminal comparisons is acceptable and don't need to be reconsidered.

\section{Conclusion}

Results according to considered criteria in this research showed that economic factor was the most important factor in creating business clusters in Choram County. Comparison of economic sub-criteria showed that demand for rural production in national and local markets was the most important sub criterion in economic factor. Thus one of the main activities for creating business clusters is development of markets for handmade products. Also markets of handicrafts should be supported by governmental organizations and private sectors. It is recommended that in addition to common marketing activities, e-marketing and local exhibitions should be developed. 


\title{
شناسايى و رتبهبندى عوامل مؤثر بر ايجاد خوشههاى كسبوكار روستايى در شهرستان هرام
}

\author{
مرضيه ارجاعى"، "همايون مرادنزادى"، محمد سلاورزى"، يارمحمد قاسمى" \\ ا - كارشئاسى ارشده كروه كار آفرينى و توسعه روستايي، دانشكده كشاورزى، دانشكاه ايلام، ايلام، ايران.

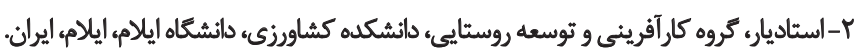

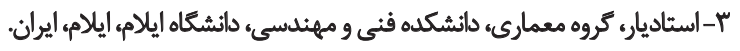 \\ F - دانشيار، كروه علوم اجتماعي، دانشكده ادبيات و علوم انساني، دانشعَاه اليلام، ايلام، ايران.
}

\begin{abstract}
حكبe

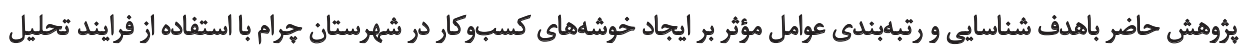

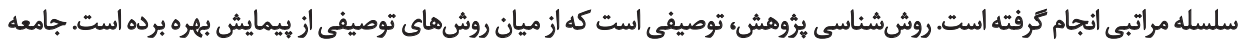

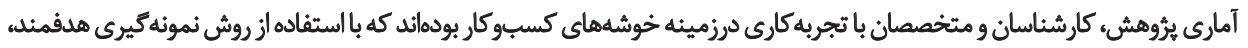

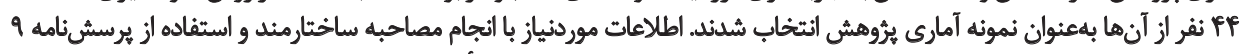

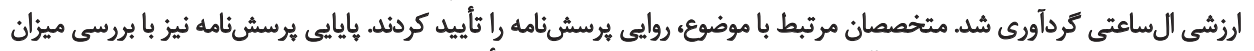

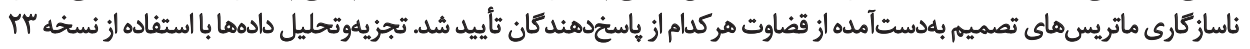

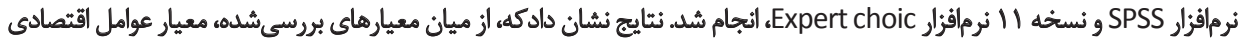

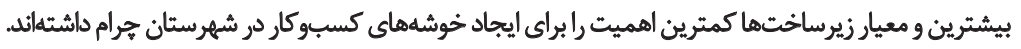

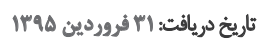

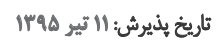

ايجاد خوشههاي كسبوكار در مناطق روستايي، ئتانسيلي براي

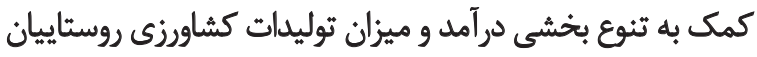

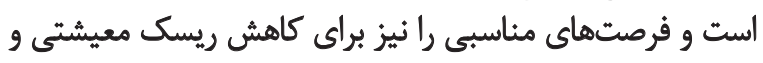

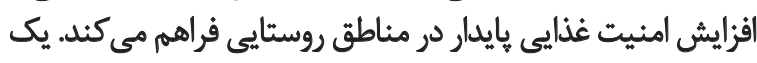

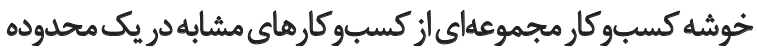
جغرافيايى است كه باعث ايجاد كسبوكارهاى مرتبط و مكمل بالي

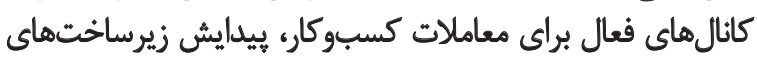

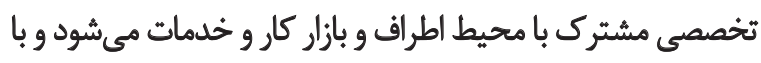

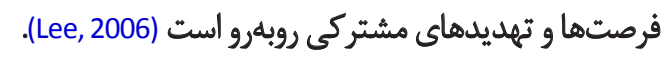

در كشور مائيز، دولت در سال هاى اخير به خوشههاى كسبوكار

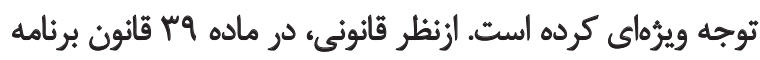

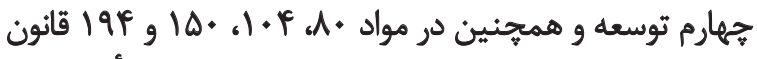

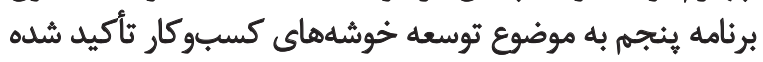

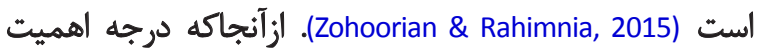

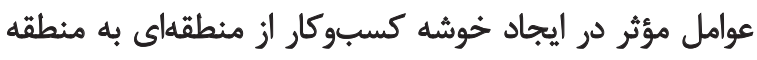

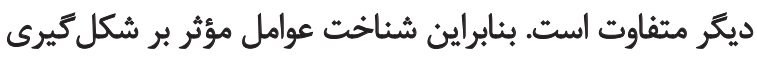

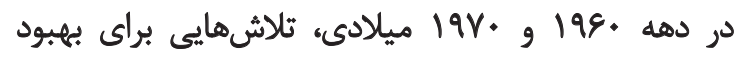
سطح زندكى روستاييان از طريق اعمال سياستهاي ارتقاي توني

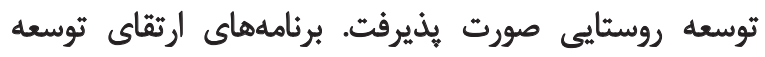

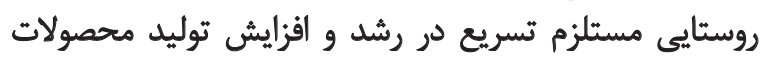

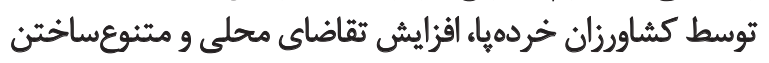

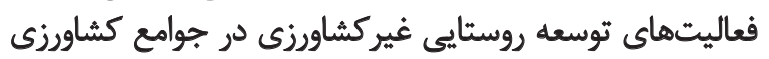
است (Shamsal-Dini \& Husseini, 2011).

نواجي روستايى بهدليل شرايط خاص بوهي و اقتصادى و مشئي

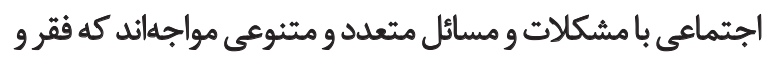

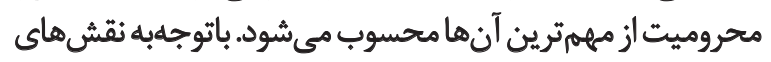

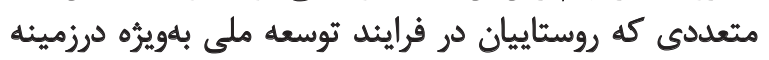

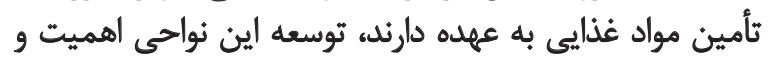

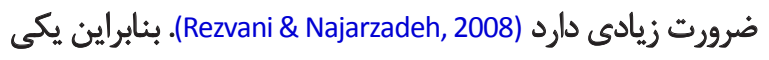

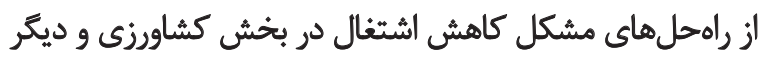

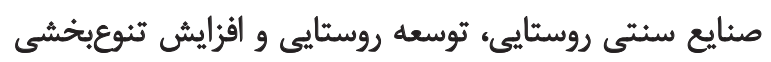

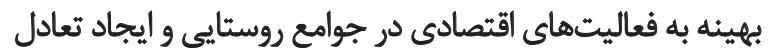


همواره يكى از مههمترين روشها براى توسعه كسبوكار و ايجاد

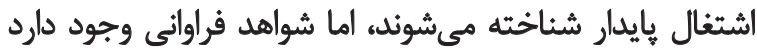

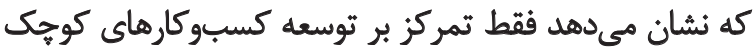

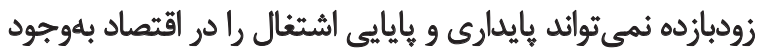
آورد، بلكه بايد از مفهوم خوشهها نيز استفادي بادئ كرد.

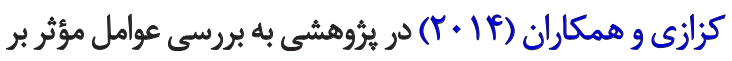

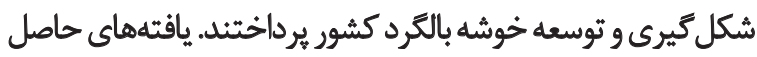

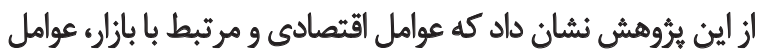

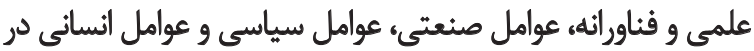

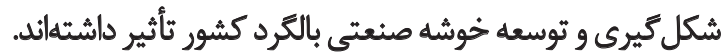

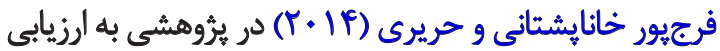

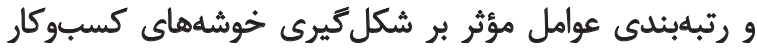

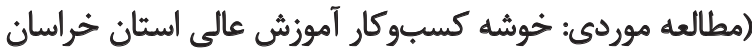

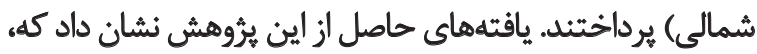

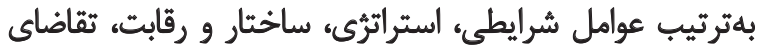

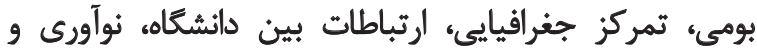

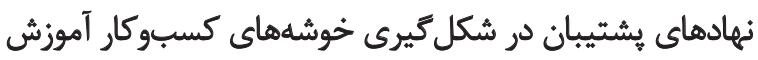
عالى استان خراسان شمالى تاثير كذار هستئد.

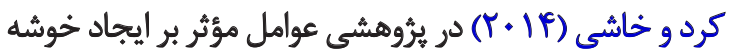

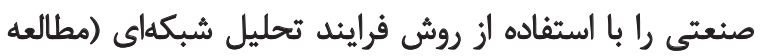

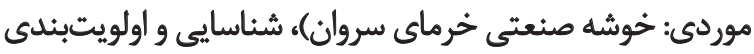

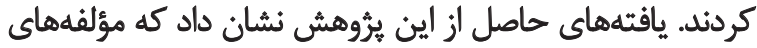

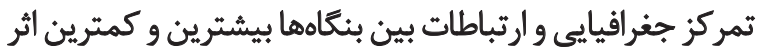

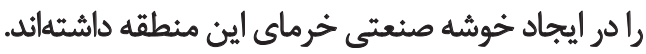

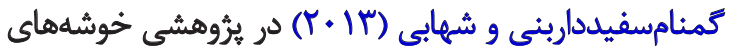

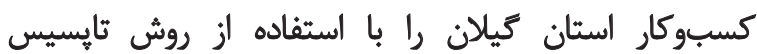

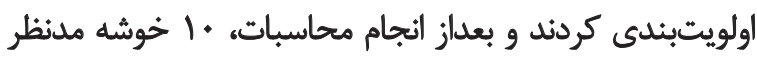

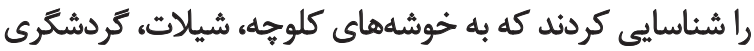

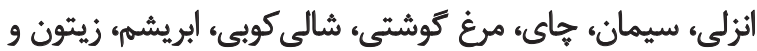

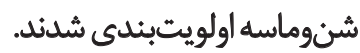

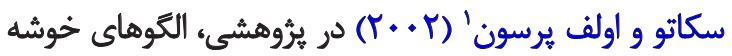

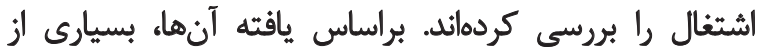

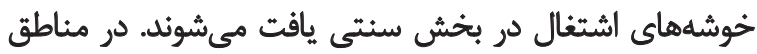

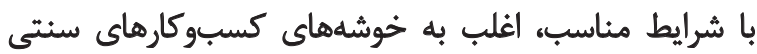

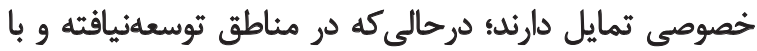

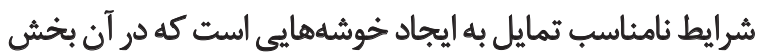
دولتى مسئول اشتغال است.

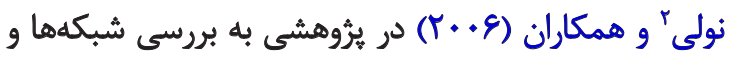

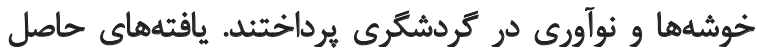

\footnotetext{
1. Ceccato \& Olof Persson

2. Novelli
}

خوشههاى كسبوكار در هرمنطقه، از نيازهاي اساسى و بسيار

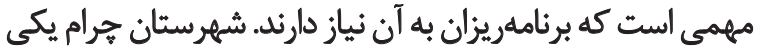

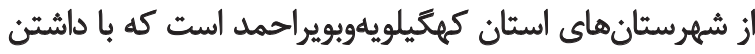

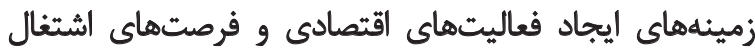

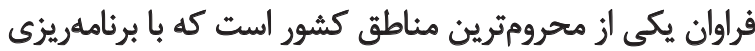

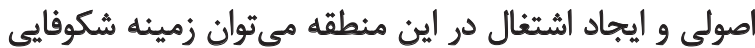
همهجانبه آن (ازنظر كشاورزى، صنعت، خدمات و و كردشكّرى)

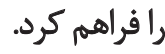

توانمندىهاو مزيتهاى موجود دراين شهرستان حاكى ازوجودي

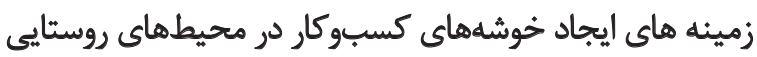

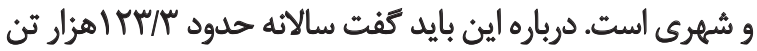

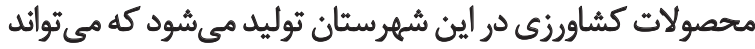

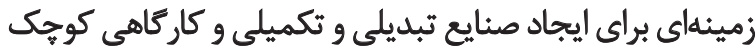

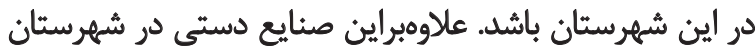

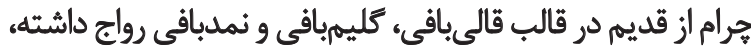

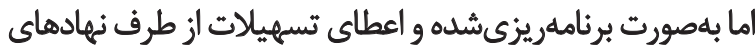

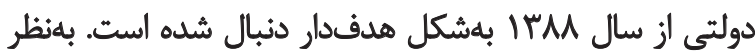

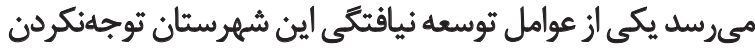
به خوشه كسبوكار است. در اين راستا با افزايش توليد سرانه، افزايش سطح اشتغال،

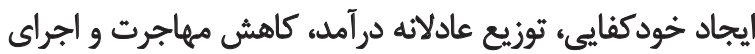

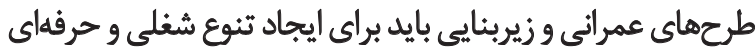

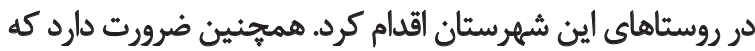

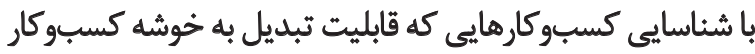

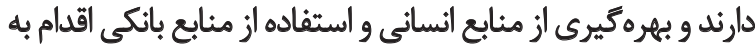

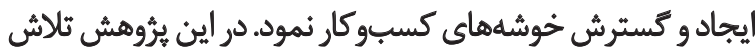

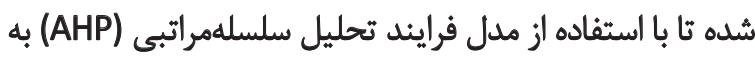

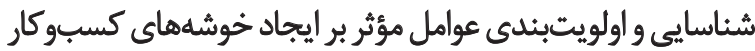

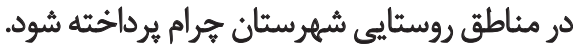

\section{T. مرورى بر ادبيات موضوع}

در سالهاى اخير هرجيند يرُوهشهايى درزمينه خوشهامها

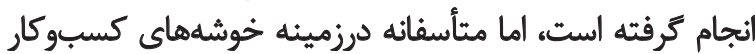

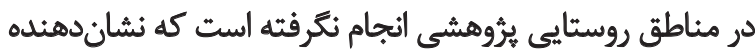

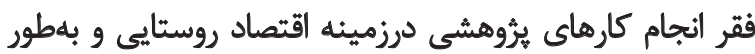

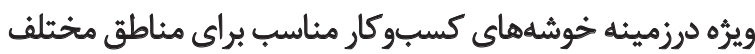

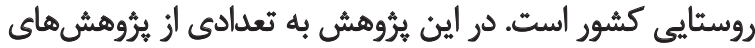

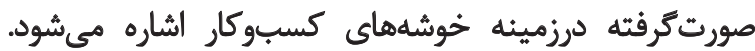

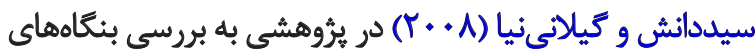

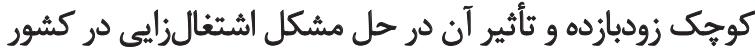

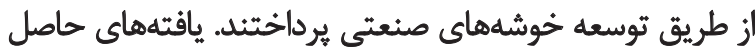

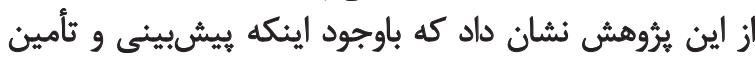

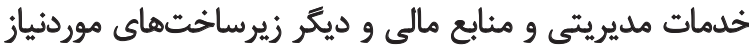




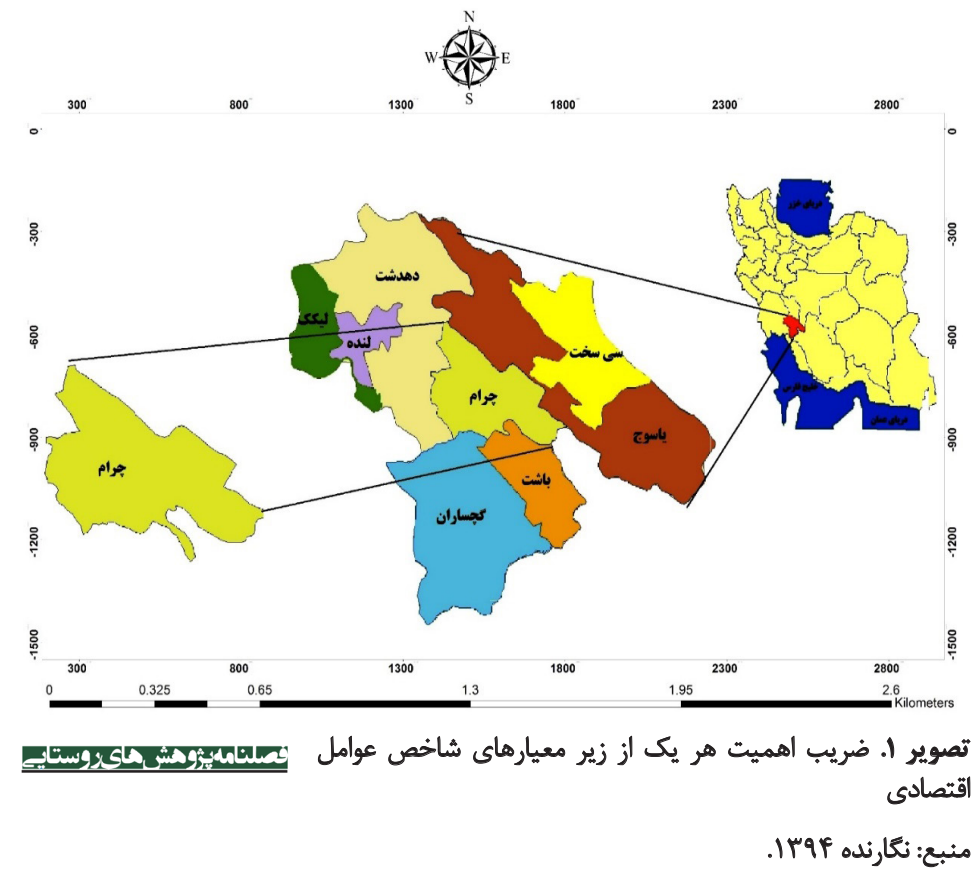

ايجاد كسبوكارهاى مرتبط مىشود و به بيدايش خدمات

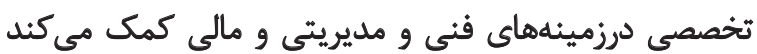
.(Delangizan, 2006)

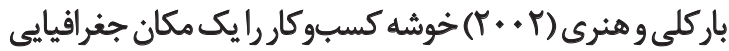

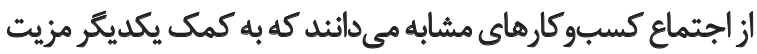

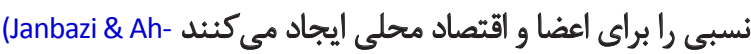
و.madi, 2008)

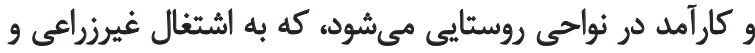

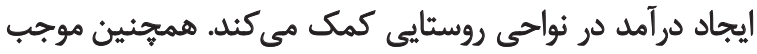

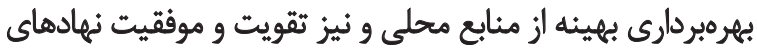

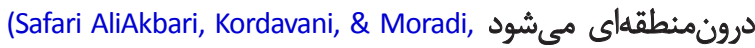

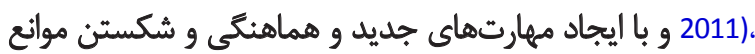

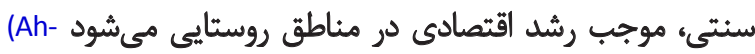
madiyan, Ghanbari, Aslani Sangdeh, \& Azizy, 2013).

اين امر مي تواند به ايجاد اشتغال محلى كمك شاياني كند و واني

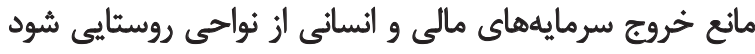
(Motiei Langroodi \& Ardeshir, 2007)

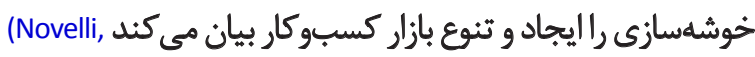

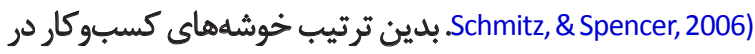

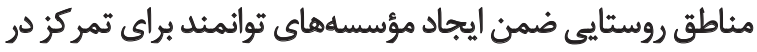

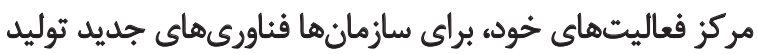

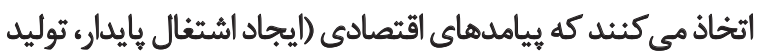

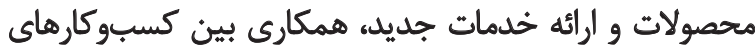

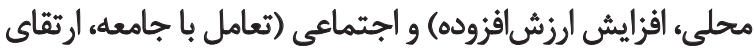

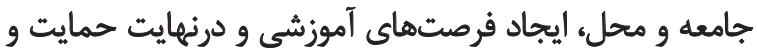

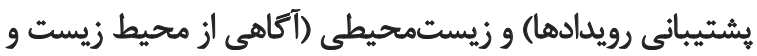

از اين يُوهش نشان داد كه همكارى بين مقامات محلى و

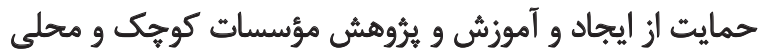
عاملى كليدى در تسهيل

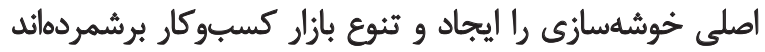

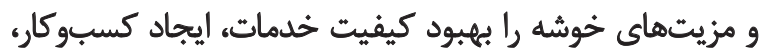

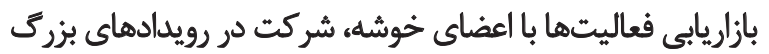

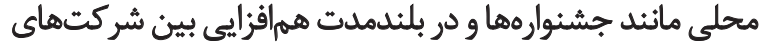

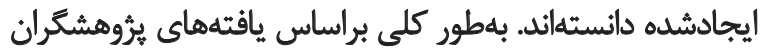

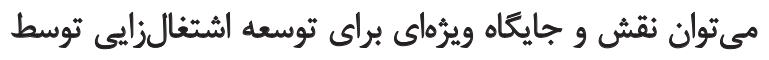

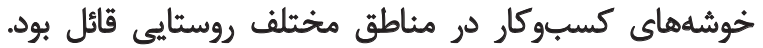

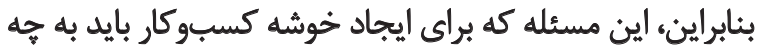

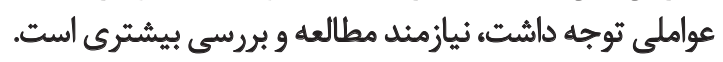

$$
\text { مبانى نظرى تحقيق }
$$

براي اولينبار مايكل يورتر (•199) در كتابي با عنوان "امزيت

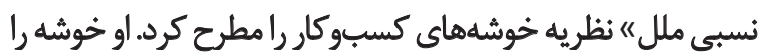

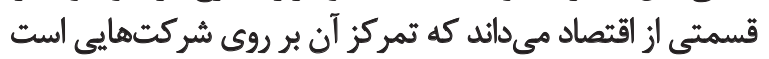

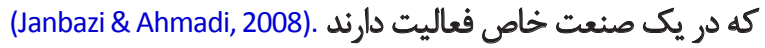

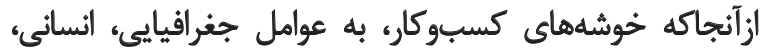

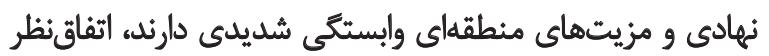

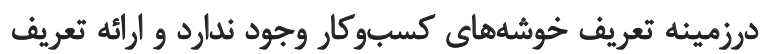
جامع و فراكير از آن مشكل است (Esfandiari \& Sacchiyan, 2009). در يك تعريف، خوشه مجموعهاى از شركتهاست كه در ناحيه

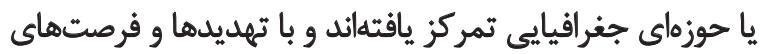

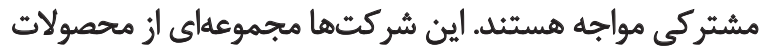

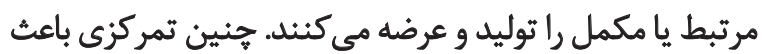


است و در ادامه فرايند تحليل سلسلهمراتبي تشريح شده است.

$$
\text { ويثَّى هاى فردى ياستخدهندكَان }
$$

براساس اطلاعات كردآ ورى شده، ميانكين سنى ياسخدهندكان

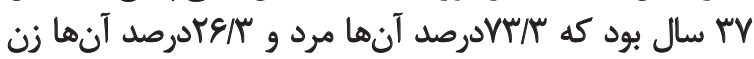

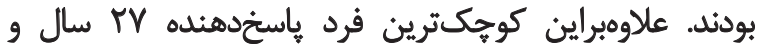

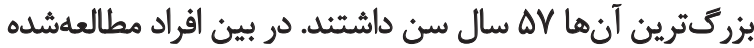

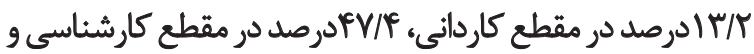

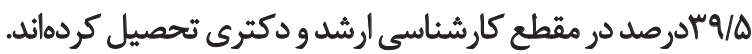

$$
\text { تعيين ساخثتار سلسلهمراتبى مسئله بررسى }
$$

هدف از انجام اين يُروهش، شناسايي و رتبهبندى عوامل مؤثر

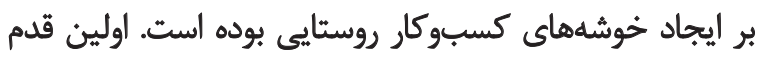

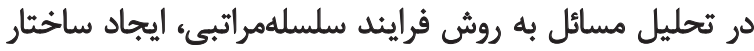

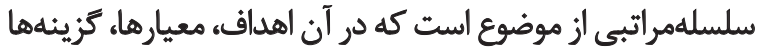

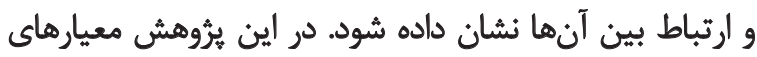

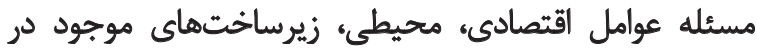

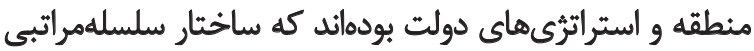
آنها در تصوير شماره ب آمده است.

تعيين ضريب الهميث زير معيار ها نسبتبه معيار

براى تعيين ضريب اهميت زيرمعيارها نسبتبه معيار، بايد وزن

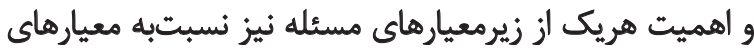

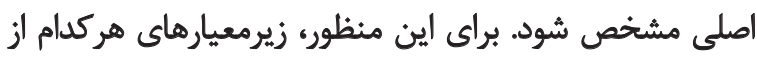

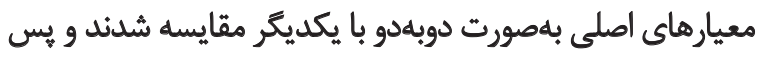

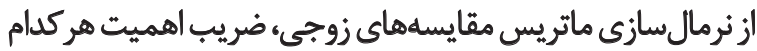

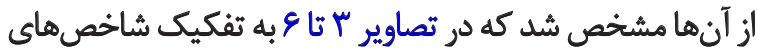

$$
\text { الصلى نشان داده شده است. }
$$

تصوير شماره ب نشاندهنده وزن زيرمعيارها باتوجهبه معيار

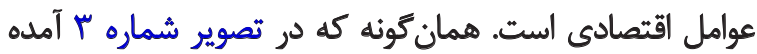

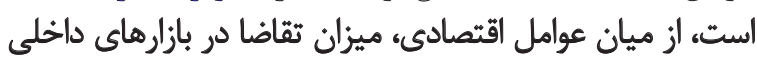

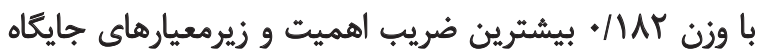

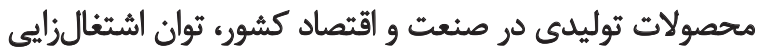

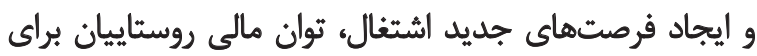

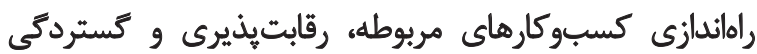

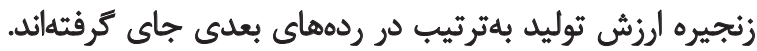

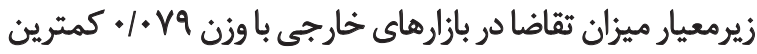

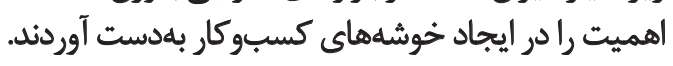

به اين ترثيب، از ميان زيرمعيارهاى عوامل اقتصادى ميزان

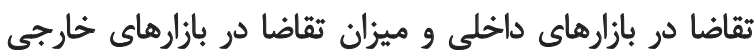

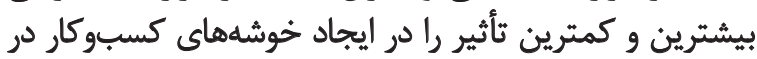

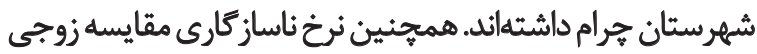

حفاظت از آن) دارند (Steiner \& Atterton, 2015).

با وجود اين هنوز هيج درك كاملى از تركيب و تكامل اقتصاد

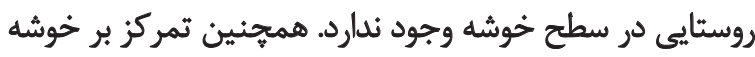

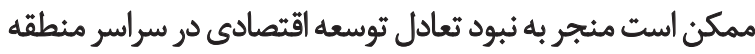
يا در بخشى از جمعيت شود (Shields, \& Barkley \& Emery, 2009)

$$
\text { قلمرو جغرافيايى تحقيق }
$$

اين يُروهش ازلحاظ جغرافيايي، محدوده شهرستان جرام استان

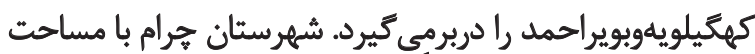

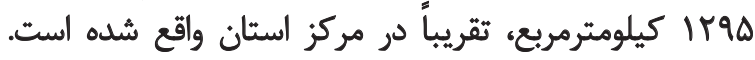

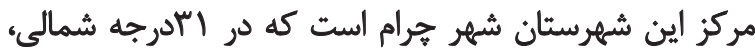

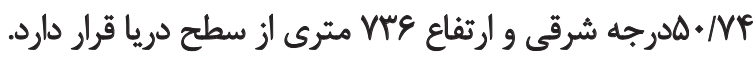

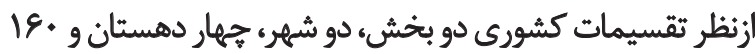

آبادى دارد (تصوير شماره () (Statistical Center of Iran, 2014)

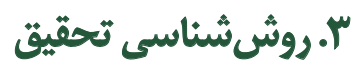

اين يُروهش ازنظر هدف كاربردى، ازنظر ميزان و درجه كنترل

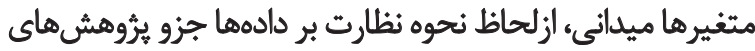

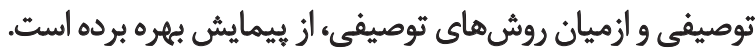

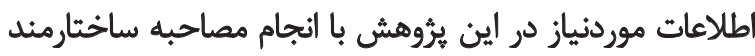

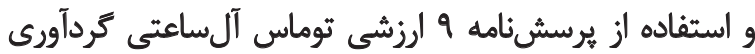

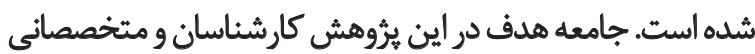

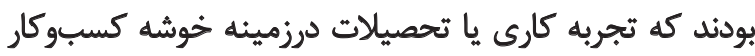

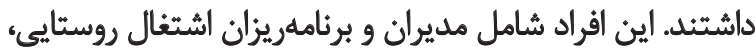

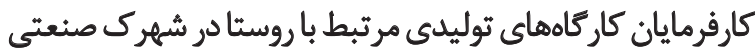

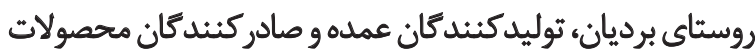
توليدى در مناطق روستايى منطقه بودند.

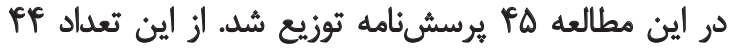

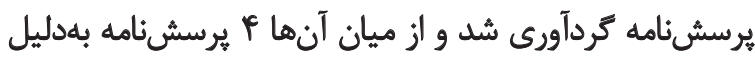

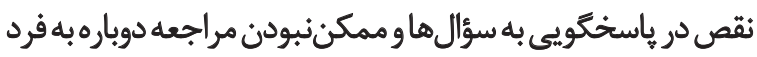

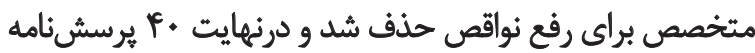

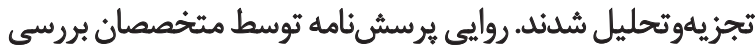

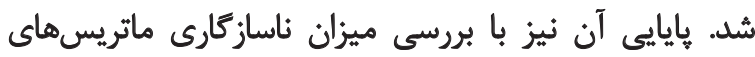

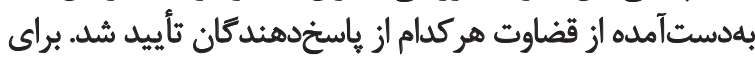

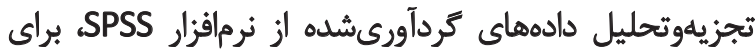

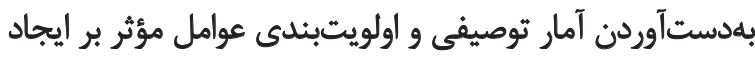

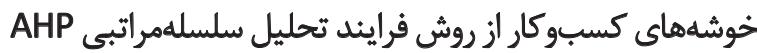

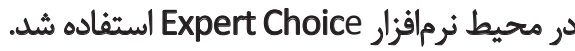

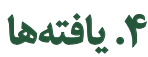

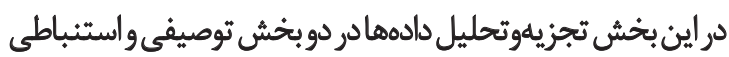

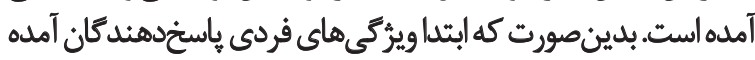




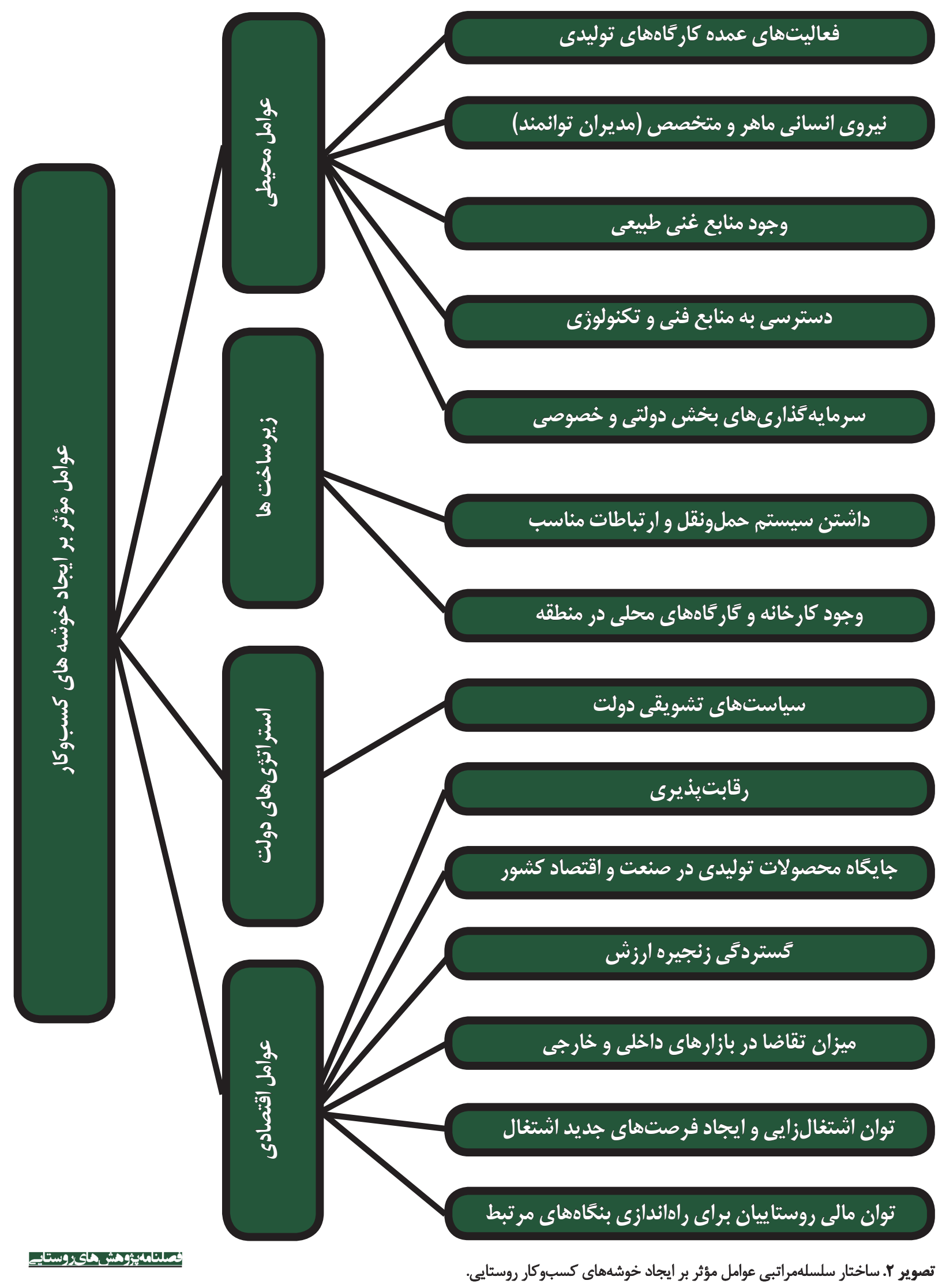




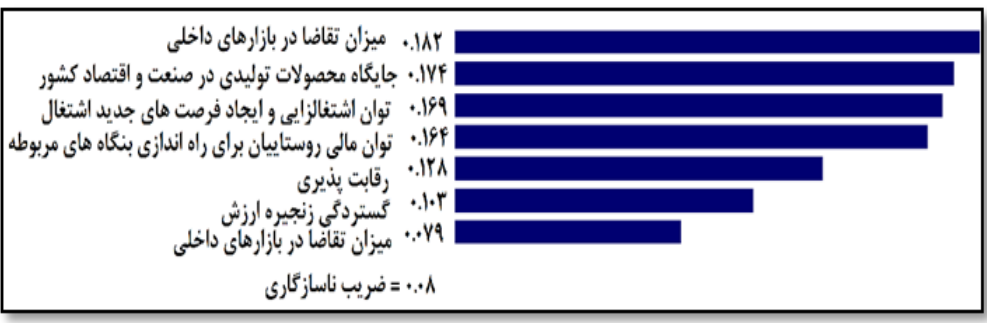

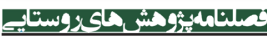

تصوير "r. ضريب اهميت هريك از زيرمعيارهاى شاخص عوامل اقتصادى.

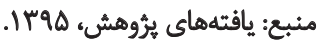

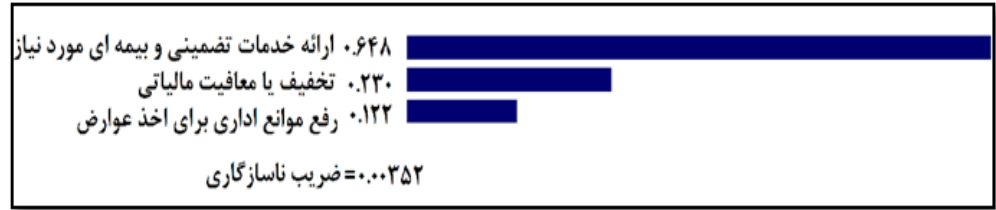

每

تصوير F. ضريب اهميت هريك از زيرمعيار هاى شاخص استراترثى هاى دولت.

منبع: يافتههاى بثوهش، هوب 11.

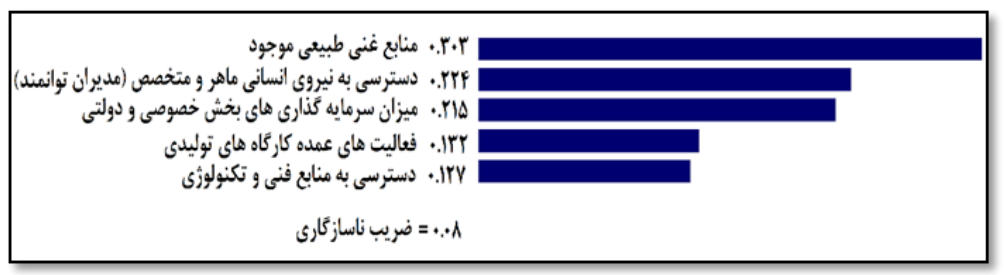

:

تصوير ه. ضريب اهميت هريك از زيرمعيارهاى شاخص عوامل محيطى.

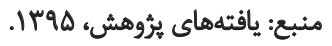

ميان زيرمعيارهاى مربوط به استرائزى هاى دولت در تشكيل

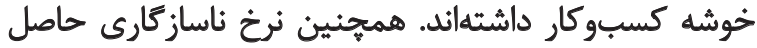

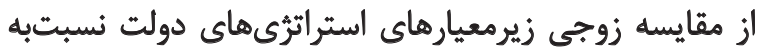

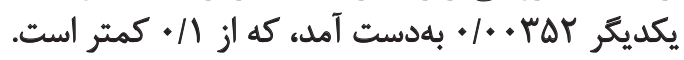

تصوير شماره ه هشان دهنده اولويتبندي زيرمعيار هاي عوامل

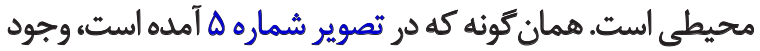

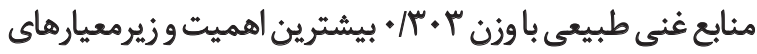
دسترسى به نيروى انسانى ماهر و متخصص باني (مديران توانمند)،
زيرمعيار هاى عوامل اقتصادى نسبتبه يكديكر 1 • ٪ بهدست آمد

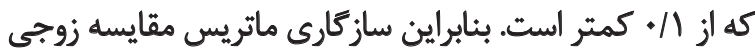
قابل قبول بود و نيازى به تجديدنظر نبون تصوير شماره أنشاندهنده اولويتبندى زيرمعيارهاي

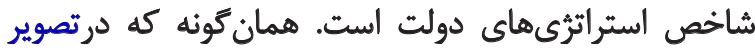

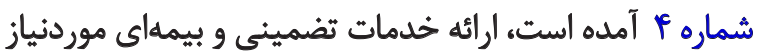

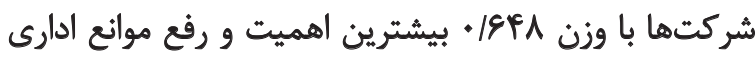

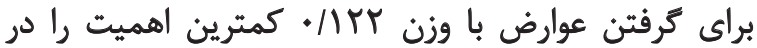

$$
\begin{aligned}
& \text { و9YY }
\end{aligned}
$$

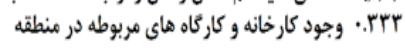

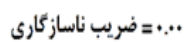

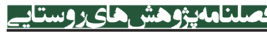

$$
\text { تصوير و. ضريب اهميت هريك از زيرمعيارهاى شاخص زيرساخت }
$$




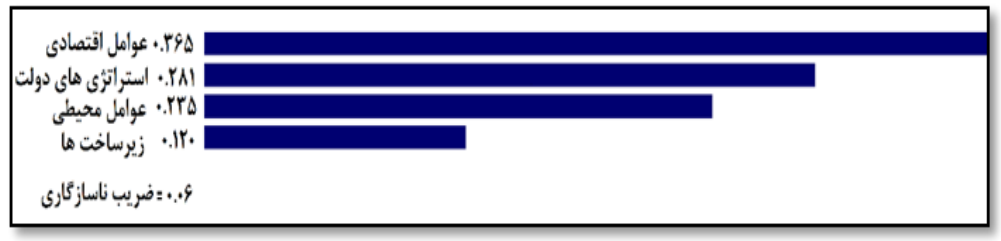

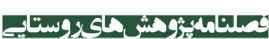

تصوير V. ضريب اهميت معيارهاى مؤثر بر ايجاد خوشه كسبوكار.

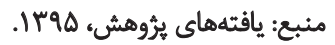

يس از نرمال سازى ماتريس هاى بهدستآمده، ميانكين هرسطر

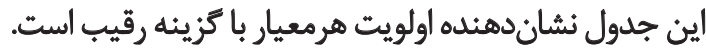

در اين هئوهش، مقايسههاى زوجى معيارها و زيرمعيارها

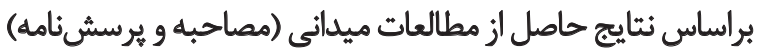

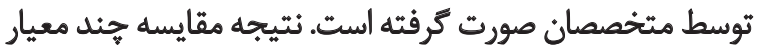

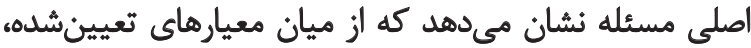

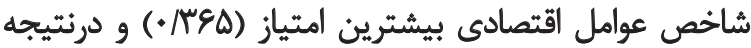

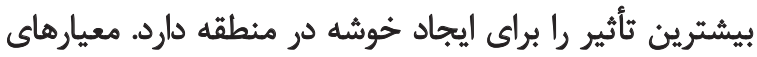

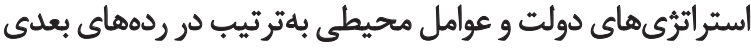

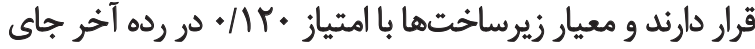

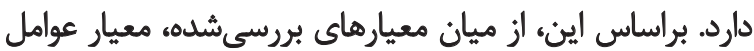

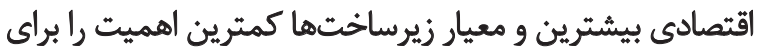

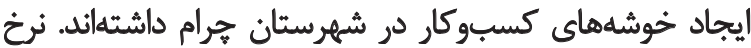

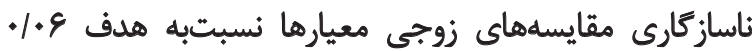

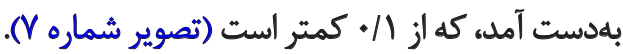

\section{م. بحث و تثيجهئيرى}

براساس نتايج بهدستآمده از تحليل سلسلهمرائبى، عوامل

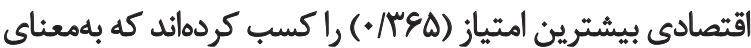

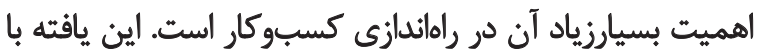

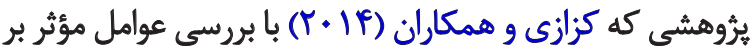

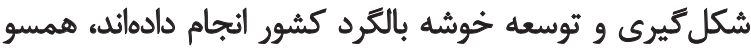

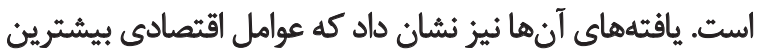

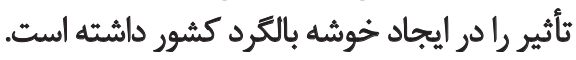

مقايسه زيرمعيارهاي عوامل اقتصادى نيز بيانكر ارجحيت

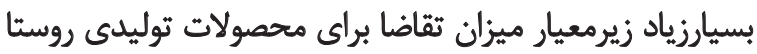

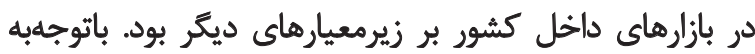

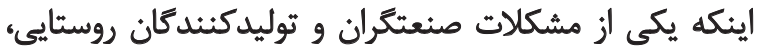

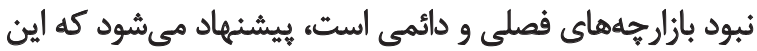

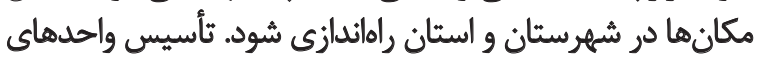

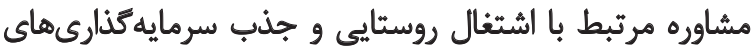

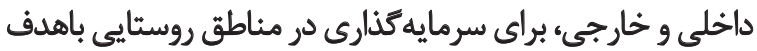

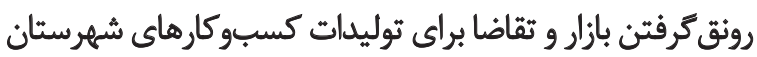

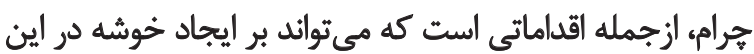

ميزان سرمايهُذارى هاى بخش خصوصى و دولتى و فعاليتهاى

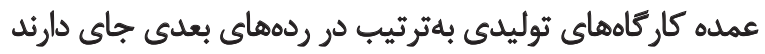

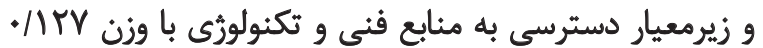

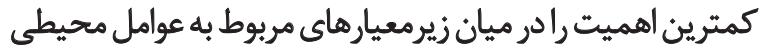

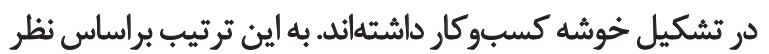

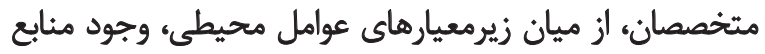

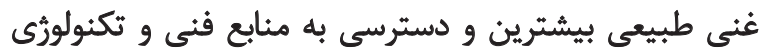

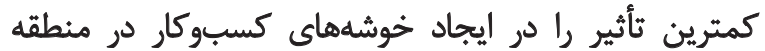

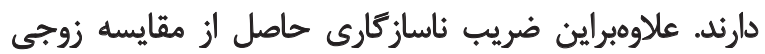

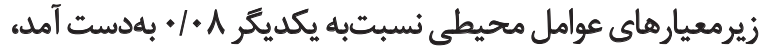

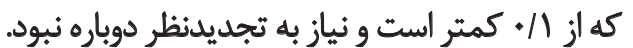
تصوير شماره \& نشاندهنده اولويتبيندى زيرمعيارهاى

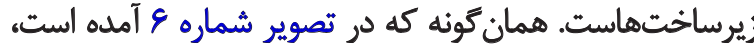

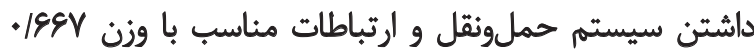

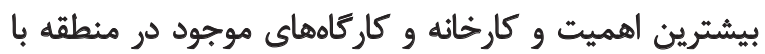

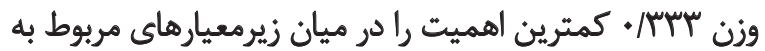
زيرساختها در تشكيل خوشه كسبوكار المديت داشتهاند.

ثعيين ضريب الهميث معيار ها نسبثبه هدف

يس از تعيين ضريب اهميت هريك از زيرهعيارها نسبتبه معيارها، بايد وزن و اهميت هريك از هي معيارهاي مسئله نيز نسبتبه هدف مشخص شود. براى تعيين ضريب اهميت هيت معيارها

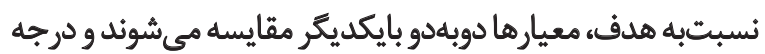

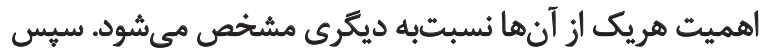

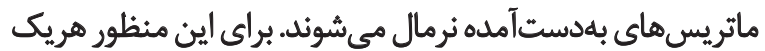

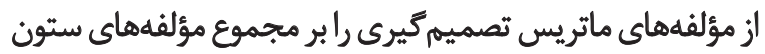

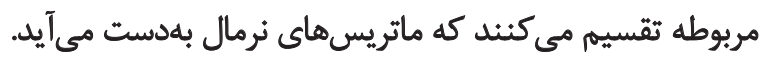
فرمول رياضي اين مطلب بلهصورت زير است:

$$
(j=1,2, \ldots, m)_{g}=\frac{a_{i j}}{\sum_{i=1}^{n} \bar{a}_{i j}} r_{i j}
$$

در اين رابطه rij معرف ارزش نرمالشده كزينه شاخص أ به شاخص ز است. 
و تعاون، كار و رفاه اجتماعي شهرستان هرام و كارفرئمايان

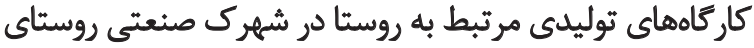

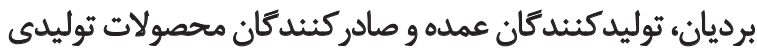
در مناطق روستايى شهرستان جرام قدردانى نماينداند
شهرستان مؤثر باشد.

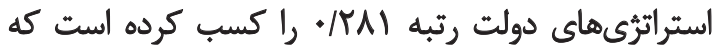

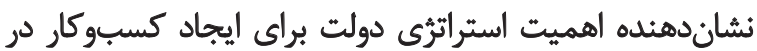

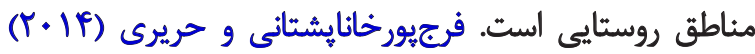

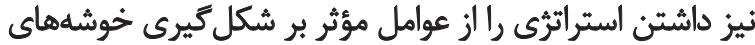

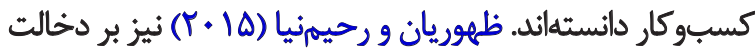
مؤثر دولت براى توسعه و ايجاد خوشهان

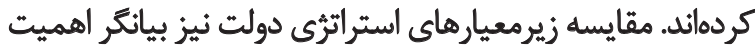

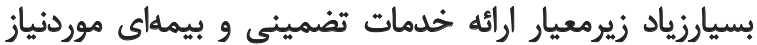

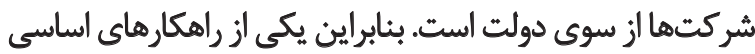

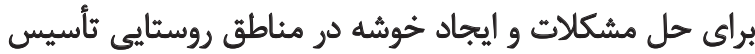

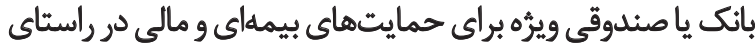

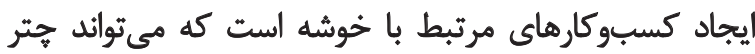

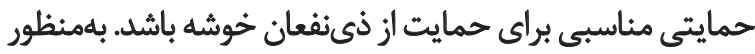

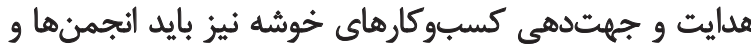
نهادهاى مرتبط با اين موضوع ايجاد شوندائ

نتايج نشان داد كه عوامل محيطى سومين عامل مهيم در

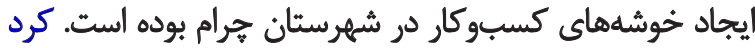

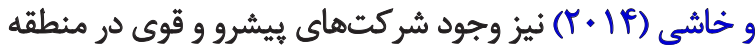

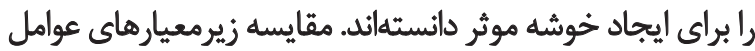

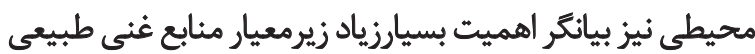

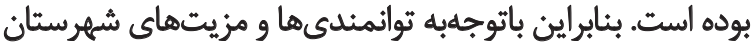

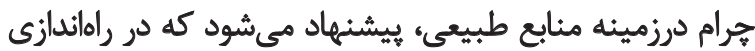

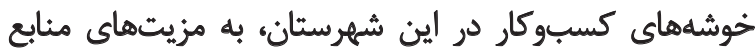
طبيعى آن توجه شود.

براساس تحليل سلسلهمراتبى متغيرهاى يُؤوهش، عوامل

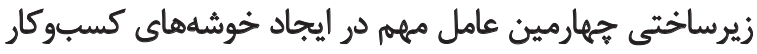

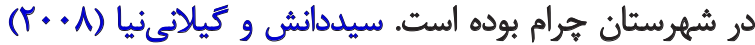

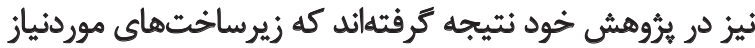

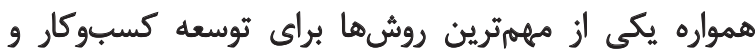

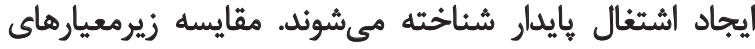

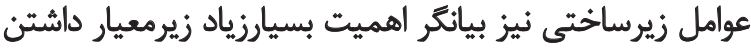

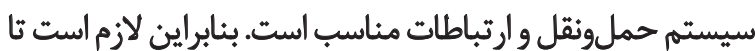

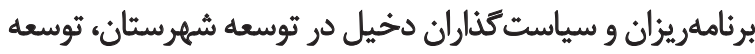

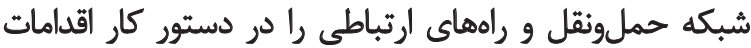
توسعه شهرستان قرار دهند.

$$
\text { تشكر و قدردانى }
$$

اين برُوهش بركرفته از يايان نامه كارشناسى ارشد خانم مرضيه

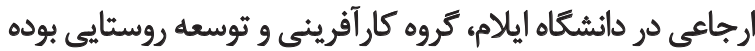

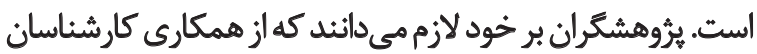

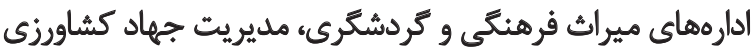




\section{References}

Ahmadian, M., Ghambari, Y., Aslani Sangade, B., Azizi, H. (2013). [Identification and analysis of factors effective in underdevelopment of agricultural conversion and complementary industries (Case study: Marvdasht County) (Persian)]. Journal of Research and Rural Planning, 4, 27-31.

Ceccato, V., \& Olof Persson, L. (2002). Dynamics of rural areas: an assessment of clusters of employment in Sweden. Journal of Rural Studies, 18(1), 46-63. doi: 10.1016/s0743-0167(01)00028-6

Delangizan, S. (2006). [Technological and science clusters, as like a General Theorem (Persian)]. Roshd-e Fanavari, 2(5), 16-25.

Esfandiari, A. A., \& Sakian, M. (2009). [Industrial chain analysis of industrial clusters (A case study of Khuzestan province) (Persian)]. Name-ye Mofid, 15(75), 129-46.

Farajpour Khanposhtani, Gh., \& Hariri, A. (2014). [Assessing and ranking the factors affecting the formation of business clusters (Case Study: Business cluster of higher education in North Khorasan Province) (Persian)]. Paper presented at the International Conference on Business Development and Excellence, Tehran, Iran, 26 December 2014.

Gomnam Sefid-Darboni, M., \& Shahabi, A. (2013). [Prioritize business clusters Gilan Province with TOPSIS (Persian)]. Paper presented at the $1^{\text {st }}$ National Conference on Engineering Business Management, Kerman, Iran, 20-21 February 2013.

Janbazi, R., \& Ahmadi, M. (2008). [The need for clustering of small and medium enterprise (SME) firms to reduce mortality (Persian)]. Paper presented at the $1^{\text {st }}$ National Conference on the Development of Economic Activity, Tehran, Iran, 23-24 December 2008.

Kazazi, A., Tabatabaeian, S. H., Amiri, M., \& Shirazi Shayesteh, M. (2014). [Analyzing factors affecting the formation and development of innovative cluster in Iranian helicopter industry (Persian)]. Innovation Management Journal, 3(1), 23-45.

Kord, B., \& Khashi, E. (2014). [Identifying and prioritizing factors affecting the creation of industrial clusters using analytic network process (Case study: Saravan dates industrial clusters) (Persian)]. Journal of Entrepreneurship Development, 7(2), 247-65.

Lee, T. (2006). Action strategies for strengthening industrial clusters in southern Taiwan. Technology in Society, 28(4), 533-52. doi: 10.1016/j.techsoc.2006.09.007

Motiei Langroodi, H., \& Ardeshir, A. (2007). [The study of Kalat's water resource with an emphasis on the problem of providing rural water (Persian)]. Geographical Research, 40(2), 1-15.

Novelli, M., Schmitz, B., \& Spencer, T. (2006). Networks, clusters and innovation in tourism: A UK experience. Tourism Management, 27(6), 1141-152. doi: 10.1016/j.tourman.2005.11.011

Rezvani, M. R., \& Najarzadeh, M. (2008). [Villagers in the process of examining and analyzing the areas of entrepreneurship development in rural areas (Case study: Rural district South Bara'an, (Esfahan Township) (Persian)]. Journal of Entrepreneurship Development, 1(2), 161-82.

Rizov, M. (2005). Rural development under the European CAP: The role of diversity. Social Science Journal, 42(4), 621-628. doi: 10.1016/j.soscij.2005.09.003

Safari Ali Akbari, M., \& Kardavani, P. (2011). [Determination of private sector's investing fields for developing the rural industries activities in Kermanshah rural areas (Persian)]. Journal Of Geographical Landscape, 6(16), 12-15.

Seyed Danesh, S. Y., \& Gilaninia, S. (2008). [Small businesses solve the problem soon returns and its impact on employment in the country through the development of industrial clusters (Persian)]. Paper presented at the $1^{\text {st }}$ National Conference on the Development of Economic Activity, Tehran, Iran, 23-24 December 2008.

Shamsoddini, A., \& Hosseini, S. N. (2011). [the role of small industries cooperatives in entrepreneurship and rural development (Persian)]. Cooperation and Agriculture, 22(6), 135-57.

Shields, M., Barkley, D., \& Emery, M. (2009). Industry clusters and industry targeting. Targeting Regional Economic Development, 44, 35 .

Statistical Center of Iran. (2014). General Population and Housing Census of Kohgiluyeh \& Boyer-Ahmad Province. Tehran: Statistical Center of Iran.

Steiner, A., \& Atterton, J. (2015). Exploring the contribution of rural enterprises to local resilience. Journal of Rural Studies, 40, 30-45. doi: 10.1016/j.jrurstud.2015.05.004

Zohoorian, M., \& Rahimnia, F. (2015). [Designing a model for sustainable development of business clusters in Iran (Persian)]. Journal of Entrepreneurship Development, 8(1), 41-59. 